\title{
Qualidade do atendimento nas Unidades de Saúde da Família no município de Recife: a percepção do usuários
}

\author{
Quality of care in the family healthcare units \\ in the city of Recife: user perception
}

Renata Florêncio Santiago ${ }^{1}$

Antonio da Cruz Gouveia M endes ${ }^{2}$

Gabriella M orais Duarte M iranda ${ }^{2}$

Petra Oliveira Duarte ${ }^{2}$

Betise M ery Alencar Sousa M acau Furtado ${ }^{2}$

Wayner Vieira de Souza ${ }^{2}$

${ }^{1}$ Coordenação Geral de Acompanhamento e M onitoramento da Gestão Descentralizada, M inistério da Saúde. Esplanada dos Ministérios Bloco G. 70058-900 Brasília DF. re_fsantiago@yahoo.com.br ${ }^{2}$ Centro de Pesquisas Aggeu M agalhães CPqAM/Fiocruz.
Abstract This study seeks to assess user perception regarding the quality of carein Family $\mathrm{H}$ ealth Units in Recife. It is a descriptive cross-sectional study adopting a quantitative approach. Thesurvey was conducted in Recife and 939 users were interviewed, being predominantly young adults, female, married, housewives with low levels of education. They have strong links with the units, taking it as a benchmark for their care. The re sults show care in accordance with scheduled demand and usersmanifest marked satisfaction with the work of the professionals and less satisfaction with the conditions offered at the units. The younger and more educated the users are, theless satisfied they are with the conditions offered. There is considerable dissatisfaction with delays in attendance, in accessibility and the lack of educational and community activities, the latter two dimensions being related to the core principles of this strategy. The marked satisfaction with clinical care, confidentiality and right to information, which are dimensions related to userprofessional relationship are fundamental to the quality of care. This evaluation focused on each aspect of the service provided and makes an important contribution with the critical appraisal reported by users.

Key words Health evaluation, User satisfaction, Family Health Program
Resumo Este estudo tem por objetivo avaliar a percepção dos usuários com a qualidade do atendimento nas Unidades de Saúde da Família do Recife. Trata-se de um estudo descritivo de corte transversal com abordagem quantitativa. A pesquisa foi realizada no Recife, sendo entrevistados 939 usuários. O susuários são predominantemente adultos jovens, do sexo feminino, casados, com baixo nível de escolaridade e donas de casa. Apresentam alto vínculo com asunidades, tendo-a como referência para o seu atendimento. Os resultados evidenciam a assistência à demanda programada. Os usuários apresentam maior satisfação com 0 trabalho dos profissionais e uma menor satisfação com as condições oferecidas pelasunidades. Quanto mais jovem e com mais escolaridade, maior a insati sfação com as condições oferecidas. A presentaram grande insatisfação com o tempo de espera, com a acessibilidade e com a insuficiência de atividades educativas e comunitárias, sendo as duas últimas dimensões relacionadas aos princípios gerais desta estratégia. A grande satisfação com as sistência clínica, confidencialidade e direito a informações, dimensões relacionadas à relação profissional-usuário são determinantes na qualidade da assistência. Esta avaliação enfocando cada momento do atendimento contribuiu na sua apreensão crítica pelos usuários. Palavras-chave Avaliação em saúde, Satisfação do usuário, Programa Saúde da Família 
Introdução

0 interesse dos gestores pela avaliação da qualidadeéum fenômeno antigo, no entanto, ao longo do tempo, o foco da avaliação e os meios de realizá-la sofreram mudanças. Esse termo apresenta diversos conceitos de acordo com a nuance a qual o autor intérprete se utiliza, no entanto, é possível observar que está sempre relacionada a um julgamento de valor com o objetivo de subsidiar o planejamento e a tomada de decisões ${ }^{1,2}$.

Ao citar a importância da avaliação em saúde, Deslandes ${ }^{3}$ refere sua relevância para Saúde Pública, já que viabiliza escol has de planejamento e permite um controle técnico esocial dos serviços e programas prestados à sociedade. Peixoto ${ }^{4}$ complementa que a qualidade pode ser considerada 0 aspecto central da avaliação.

ParaD onabedian 5 a idéia dequalidadeestápre sente em todos os tipos de avaliação, uma vez que sua característica principal é o estabelecimento de um juízo, a atribuição de um valor a alguma coisa que, quando positivo, significa ter qualidade.

Este autor desenvolveu um quadro conceitual fundamental para o entendimento da avaliação da qualidade em saúde, a partir dos conceitos de estrutura, processo e resultado, classicamente considerados uma tríade. 0 componente estrutura corresponde à avaliação dos recursos existentes para a execução dos serviços; por processo entende-se a avaliação da execução das atividades e da dinâmica apresentada nas suas interrelações; e por fim, o resultado representa os efeitos ou produtos das ações realizadas pelos serviços de saúde, que modificaram a situação de saúde dos usuários ou da comunidade ${ }^{6}$.

Para M alik e Schiesari ${ }^{7}$ uma forma de agregar com mais clareza o modelo de Donabedian ao da qualidade é incorporar às suas variáveis básicas (estrutura, processo e resultado) outras características, como as técnicas, interpessoais e voltadas a "amenidades", pois assim podem dar maior concretude aos conceitos deste autor.

A curcio et al. ${ }^{8}$ complementam, referindo queo processo de avaliação não possui um modelo perfeito, que já tenha sido suficientemente testado e que possa ser aplicado de modo universal, o que aponta a necessidade de avançar com um modelo que oriente e facilite a tomada de decisões, sendo aperfeiçoado com o tempo ea partir das experiências vividas durante sua implementação.

N este âmbito surge uma proposta de avaliação de desempenho de sistema de saúde brasileiro, o Projeto de Metodologia de Avaliação do Desempenho do Sistema de Saúde Brasileiro
(PRO-ADESS), coordenada pela Fundação Oswaldo Cruz (Fiocruz) ${ }^{9}$.

Para o desempenho dos serviços de saúde o PRO-ADESS 9 define que os elementos a serem mensurados devem estar em concordância com os objetivos do sistema de saúde e, a partir de uma revisão da literatura, propõe como dimensões para esta avaliação: efetividade, acesso, eficiência, respeito aos direitos das pessoas, aceitabilidade, continuidade, adequação e segurança.

Percebe-se assim, como refere Felisberto ${ }^{10}$, que a avaliação de políticas e programas de saúde deve contemplar ampla participação e o uso de múltiplos focos e métodos, permitindo que a visão de diferentes grupos seja considerada no objeto de estudo.

Assim, o modo como os cuidados técnicos são dispensadosou recebidos, no relacionamento usuá rio/serviço desaúde, podeser um importanteíndice para avaliar a qualidade da assistência ${ }^{11}$.

Para Donabedian ${ }^{6}$ na medida em que o processo de trabalho do setor saúde implica necessariamente a efetiva partici pação do usuário para a obtenção de um produto, torna-se claro que não é possível a avaliação do processo sem o envolvimento desteusuário, a quem se destina cada atividade do processo de forma imediata durante a execução.

O conceito social, como princípio do SUS, implica a possibilidade dos usuários intervirem nos serviços de saúde, tanto na proposição de políticas eações quanto na finalização de sua efe tivação. N este sentido, seu fortalecimento no âmbito do SUS e o incentivo à participação comunitária, em particular na atenção básica à saúde, pressupõem a concepção do usuário como corresponsável pela gestão do sistema de saúdee com competência para avaliá-lo, bem como para neleintervir emodificá-lo ${ }^{11}$.

Desta forma, a avaliação da percepção dos usuários em relação à qualidade do atendimento nas Unidades de Saúde da Família (USF) no município do Recifeapresentagrande relevância, uma vez quea aval iação do sistema de saúde pelo usuário favorece a humanização do serviço, além de constituir uma oportunidade de se verificar, na prática, a resposta da comunidade à oferta do serviço de saúde, como também permitir a adequação do mesmo às suas expectativas.

\section{Metodologia}

Trata-se de um estudo descritivo de corte transversal, que analisou um fenômeno social queéa 
qualidade da assistência prestada, sendo desenvolvido dentro de uma abordagem quantitativa.

Em 2008, a rede ambulatorial básica de saúde na Cidade do Recife era composta por 24 Unidades Básicas Tradicionais (Centros deSaúde) e112 Unidades e 240 Equipes de Saúde da Família, com uma cobertura de 54\% do Programa de Saúde da Família, distribuídas de acordo com a necessidade da população nos seis distritos sanitários.

Para fins deste estudo, definiu-se que as entrevistas seriam realizadas com os usuários que foram atendidos pelo médico e pelo enfermeiro das Equipes de Saúde da Família (ESF).

Para cálculo da amostra da população de estudo, foi mensurado o potencial de atendimento semanal dos médicos e enfermeiros das USF do município. Sendo assim, considerando que estes profissionais atendem entre 5 a 7 turnos semanais, com cota deatendimento de 16 e 12 pacientes por turno, respectivamente, econsiderando 0 potencial de $80 \%$ desta capacidade instalada semanal, calculou-se uma meta total de 32.256 atendimentos por semana. Por fim, para garantir a representatividade da amostra e considerando todas as possibilidades para a viabilidade do estudo, assim como a natureza e as particularidades da população, definiu-se por amostrar 960 usuários o que equivale a aproximadamente 3\% da capacidade de atendimento das USF. Tal fração amostral garantepoder de aproximadamente 96\% para diferenças entre proporções na ordem de 0,1 .

Essa amostra foi distribuída proporcionalmente nas unidades tomando como base de estratos, os Distritos Sanitários. Como as unidades de saú de da família são subdivididas em equipes e definindo uma fração amostral de $20 \%$ do universo de equipes, teve-se um tamanho amostral de48ESF, que foram distribuídas nos Distritos Sanitários, considerando a proporção decada distrito com relação ao universo total de equipes. Assim, com a definição do número de usuários participantes (960), e o número de equipes a serem estudadas (48), obteve-se uma média de 20 usuários para cada ESF, distribuídos da seguinte forma: 10 usuários atendidos pelo médico e 10 atendidos pelo enfermeiro. AsESF foram sorteadas de forma al eatória dentretodas as equipes de cada distrito sanitário.

A coleta de dados foi realizada por oito bolsistas, estudantes do último ano do curso de enfermagem da Universidadede Pernambuco (UPE). Optou-se pela seleção destes, pela sua familiaridade com a Política Nacional de Atenção Básica e aEstratégia de Saúde da Família facilitando assim a compreensão das questões postas aos entrevistados, bem como a familiaridade com a metodologia científica. Os estudantes passaram por um treinamento para conhecer os objetivos da pesquisa e o instrumento a ser aplicado.

O questionário foi elaborado especificamente para os propósitos desta pesquisa. E para tanto, houve a necessidade de definição das dimensões da qualidade da assistência a serem estudadas.

Para definição destas dimensões teve-se como referência a análise de estudos sobre indicadores, dimensões e propostas de qualificação da atenção à saúde real izado pela M etodologia deAvaliação do Desempenho do Sistema de Saúde (PROADESS) ${ }^{9}$, levando em consideração a priorização da avaliação da relação interpessoal, no âmbito da atenção básica: confidencialidade das informações; direito à informação; conforto; dignidadee cortesia; acessibilidade; assistência clínica; tempos de espera; desenvolvimento de atividades comunitárias.

Outra abordagem da qualidade utilizada neste estudo buscou como referencial teórico a proposta de Donabedian ${ }^{6}$, que propõe que as avaliações em saúde tenham como eixos para sua realização as análises de estrutura, processo e resultado. Desta forma, esta pesquisa estudou um dos resultados na atenção à saúde que é a satisfação dos usuários através da avaliação da estrutura (condições oferecidas pelas unidades de saúde) e processo de trabalho (ação e inter-relação humana na atenção à saúde do usuário).

Cada questão avaliada teve atribuído um valor de 0 a 10, formato baseado na escala Likert, de forma que para avaliação de cada uma das questões contidas no questionário foi feita a média por indivíduo e para avaliação das categorias foi mensurada a média das questões contidas na categoria.

N os estudos de satisfação, apesar da diversidade de métodos e instrumentos, a satisfação é em geral estabelecida como boa ou muito boa; alta ou muito alta12,13 ou quando a avaliação obtém uma nota igual ou superior a sete ${ }^{14}$. Desta forma, considerando que a média geral das notas obtidas nesteestudo foi 7,03, definiu-secomo satisfação do usuário com 0 atendimento das unidades de saúde da família, as avaliações com notas iguais ou superiores a sete. Para verificar a existência de diferenças significativas na distribuição das frequências entre insatisfeitos e satisfeitos, utilizou-se o teste de Qui-quadrado de Tendência Linear, queavalia a relação linear entre as duas variáveis e pode ser aplicado quando a variável resposta é dicotômica ea variável expo- 
sição é quantitativa ou ordinal1 ${ }^{15}$. Para todos os testes, o nível de significância adotado foi de $5 \%$.

Esta pesquisa foi aprovada pelo Comitê de Ética e Pesquisas em Seres Humanos do Centro de Pesquisas Aggeu M agalhães. Para a aplicação dos questionários foi apresentado aos entrevistados o Termo de Consentimento Livre e Esclarecido, contendo os objetivos da pesquisa e esclarecimentos quanto à participação voluntária, à garantia do direito de não identificação como também à possibilidade de a qualquer momento não mais fazer parte da pesquisa, não existindo assim, a possibilidade de danos ou riscos à população envolvida.

\section{Resultados}

Foram entrevistados 939 usuários (Tabela 1), dos quais $74,8 \%$ eram pacientes e os demais acomde Saúde da Família segundo atributos pessoais. Recife, 2009.

\begin{tabular}{lrr}
\hline \multicolumn{1}{c}{ Caracterização dos Usuários } & N & $\%$ \\
\hline Respondente & & \\
Paciente no momento & 699 & 74,8 \\
Acompanhante no momento & 236 & 25,2 \\
Profissional do Atendimento & & \\
$\quad$ Enfermeiro & 465 & 49,5 \\
M édico & 474 & 50,5 \\
Sexo & & \\
Feminino & 855 & 91,1 \\
M asculino & 84 & 8,9 \\
Faixa-etária & & \\
Até 19 anos (jovem) & 70 & 7,7 \\
20-39 anos (adulto jovem) & 497 & 54,6 \\
40-59 anos (adulto) & 273 & 30,0 \\
60 anos e mais (idoso) & 71 & 7,8 \\
Estado Civil & & \\
Casado/ União estável & 589 & 62,7 \\
Solteiro & 261 & 27,8 \\
Viúvo & 52 & 5,5 \\
Separado & 37 & 3,9 \\
Escolaridade & & \\
Sem escolaridade e Fundamental incompleto & 422 & 45,0 \\
Fundamental Completo e M édio incompleto & 276 & 29,5 \\
M édio completo & 219 & 23,4 \\
Superior incompleto e completo & 20 & 2,1 \\
Ocupação & & \\
Dona de casa & 465 & 49,8 \\
Sem vínculo empregatício & 238 & 25,5 \\
Com vínculo empregatício & 140 & 15,0 \\
Estudante & 62 & 6,6 \\
Empregador & 28 & 3,0 \\
& & \\
& & \\
& &
\end{tabular}

panhavam pacientes para consulta; $91,1 \%$ eram do sexo feminino e $54,6 \%$ encontravam-se na faixa de 20 a 39 anos. A maioria $(62,7 \%)$ dos usuários eram casados/união estável. Quanto à escolaridade, grande parte tinha ensino fundamental incompleto ou sem escolaridade $(45,0 \%)$. A maioria (49,8\%) eram donas de casa ou empregados sem vínculo formal $(25,5 \%)$.

$\mathrm{Na}$ avaliação do vínculo do usuário com aUSF (Tabela 2), 86,2\% citaram que sempre que apresentam um problema de saúde procuram a USF na qual estão vinculados para realização do atendimento, assim como, 95,6\% já tinham sido atendidos na unidade em outros momentos.

No tocante à estratégia que os usuários utilizaram para a marcação da consulta, observa-se que a maioria $(72,5 \%)$ realizou a marcação na própria unidade de saúde, e um percentual relevante de usuários (22,9\%) realizou a marcação através dos Agentes Comunitários de Saúde (ACS). Mais de $40 \%$ dos entrevistados esperaram até 7 dias para a realização do atendimento,

Tabela 2. Caracterização do Atendimento e Situação de Saúde dos U suários atendidos nas Unidades de Saúde da Família. Recife, 2009.

\begin{tabular}{lrr}
\hline $\begin{array}{l}\text { Situação de Saúde e Caracterização } \\
\text { do Atendimento }\end{array}$ & N & $\%$ \\
\hline $\begin{array}{lrr}\text { Procura a unidade sempre que apresenta } \\
\text { problema de saúde }\end{array}$ & \\
$\quad$ Sim & 806 & 86,2 \\
N ão & 129 & 13,8 \\
Primeira consulta na unidade & & \\
Sim & 41 & 4,4 \\
Não & 897 & 95,6 \\
M arcação de Consulta & & \\
Na unidade & 678 & 72,5 \\
Com o ACS & 214 & 22,9 \\
Outra & 43 & 4,6 \\
Tempo para a realização da consulta & & \\
N ão agendada & & \\
$\quad$ M esmo dia & 175 & 19,2 \\
Agendada & & \\
$\quad$ Até 7 dias & 398 & 43,7 \\
$\quad$ 8 a 14 dias & 100 & 11,0 \\
$\quad$ 15 a 30 dias & 207 & 22,7 \\
$\quad$ M ais de 30 dias & 30 & 3,3 \\
Nível de Saúde Auto-referido & & \\
Bom & 447 & 47,6 \\
Regular & 403 & 42,9 \\
Ruim & 89 & 9,5 \\
U so de medicamentos de forma contínua & & \\
Sim & 371 & 40,1 \\
Não & 555 & 59,9 \\
\hline & & \\
\hline
\end{tabular}


eum pouco mais que $19,0 \%$ conseguiram o acesso no mesmo dia.

O nível desaúde autorreferido dosusuários foi bom ou regular para $90,5 \%$ dos entrevistados e $59,9 \%$ dos usuários relataram não tomar nenhum medicamento de uso contínuo no momento.

A avaliação dos usuários com a assistência prestada demonstra que a satisfação com o trabalho dos profissionais (75,5\%) é superior à satisfação com as condições oferecidas $(41,7 \%)$ nas Unidades de Saúde da Família (Tabela 3).

A baixa satisfação com as condições oferecidas aos usuários teve diferenças estatisticamente significativas em relação às suas faixas-etárias, quanto mais jovem maior a insatisfação. Já a alta satisfação com o trabal ho dos profissionais, acima de $70 \%$, esteve presente em todas as faixas etárias, sendo ainda maior entre os usuários com 60 ou mais anos (87,3\%), porém o teste de tendência linear não revelou significância estatística (Tabela 3).

$\mathrm{Na}$ avaliação das condições oferecidas pelo PSF em relação ao nível de escolaridade (Tabela 4) observa-se que quanto maior a escolaridade menor a satisfação do usuário, os entrevistados com ensino superior incompleto e completo apresentaram quase $50 \%$ mais possibilidades de estarem insatisfeitos que os indivíduos sem escolaridade e com fundamental incompleto, num nível de significância de 10\%. Q uanto à relação en- tre satisfação com o trabalho e a escolaridade, observa-se uma alta satisfação em todos os níveis, embora seja menor entre os usuários com escolaridade superior.

A satisfação dos usuários também foi avaliada segundo as dimensões da qualidade do atendimento prestado nas Unidades de Saú de da Família do Recife, definidas para este estudo. Observou-se, uma alta satisfação, superior a $90 \%$, nas questões relacionadas às dimensões do direito e confidencialidade das informações, assim como, com a assistência clínica oferecida ao usuário (Tabela 5). Os tempos de espera, o desenvolvimento de atividades educativas e comunitárias, o conforto e a acessibilidade tiveram a mais alta insatisfação.

Aprofundando os resultados de cada uma das dimensões e as questões que a compõem, foi observado que a acessibilidade teve, na distância da casa para a unidade de saúde, a questão meIhor avaliada, com satisfação de 80,4\%, embora tenham apresentado grande insatisfação para agendar uma consulta especializada $(47,5 \%)$ e para realizar a consulta na unidade $(45,3 \%)$.

Q uanto à dignidade cortesia no atendimento, a questão com mai or satisfação foi a gentileza e o respeito dos profissionais durante 0 atendimento $(82,0 \%)$. A maior insatisfação $(63,0 \%)$ dos usuários foi com a possibilidade de fazer reclamações sobre a unidade e ser ouvido.

Tabela 3. Avaliação da satisfação dos usuários com as condições oferecidas eo trabal ho dos profissionais, segundo faixa etária dos usuários, no PSF do município do Recife, 2009.

\begin{tabular}{|c|c|c|c|c|c|c|}
\hline \multirow[b]{2}{*}{ Dimensões } & \multicolumn{2}{|c|}{ Insatisfeito } & \multicolumn{2}{|c|}{ Satisfeito } & \multirow[b]{2}{*}{ OR } & \multirow{2}{*}{$\begin{array}{c}\chi^{2} \text { Tendência } \\
\text { P-valor* }\end{array}$} \\
\hline & $\mathrm{N}$ & $\%$ & $\mathrm{~N}$ & $\%$ & & \\
\hline \multicolumn{7}{|l|}{ Condições Oferecidas } \\
\hline \multicolumn{7}{|l|}{ Faixa etária } \\
\hline Até 19 anos & 47 & 67,1 & 23 & 32,8 & 1,0 & \\
\hline 20 a 39 anos & 312 & 62,8 & 185 & 37,2 & 0,83 & \\
\hline 40 a 59 anos & 146 & 53,5 & 127 & 46,5 & 0,56 & \\
\hline 60 anos ou mais & 26 & 36,6 & 45 & 63,4 & 0,28 & \\
\hline Total & 531 & 58,3 & 380 & 41,7 & & $\begin{array}{c}\chi^{2}=20,77 \\
p=0,00001\end{array}$ \\
\hline \multicolumn{7}{|c|}{ Trabalho dos Profissionais } \\
\hline \multicolumn{7}{|c|}{ Faixa etária } \\
\hline Até 19 anos & 15 & 21,4 & 55 & 78,6 & 1,0 & \\
\hline 20 a 39 anos & 134 & 27,0 & 363 & 73,0 & 1,35 & \\
\hline 40 a 59 anos & 65 & 23,8 & 208 & 76,2 & 1,15 & \\
\hline 60 anos ou mais & 9 & 12,7 & 62 & 87,3 & 0,53 & \\
\hline Total & 223 & 24,5 & 688 & 75,5 & & $\begin{array}{l}\chi^{2}=2,95 \\
p=0,086\end{array}$ \\
\hline
\end{tabular}

${ }^{*}$ P referente ao teste qui-quadrado de Tendência e $5 \%$ de significância 
Tabel a 4. Avaliação da satisfação dos usuários com as condições ofereci das e o trabal ho dos profissionais, segundo escolaridade dos usuários, no PSF do município do Recife, 2009.

\begin{tabular}{|c|c|c|c|c|c|c|}
\hline \multirow[b]{2}{*}{ Dimensões } & \multicolumn{2}{|c|}{ Insatisfeito } & \multicolumn{2}{|c|}{ Satisfeito } & \multirow[b]{2}{*}{ OR } & \multirow{2}{*}{$\begin{array}{l}\chi^{2} \text { Tendência } \\
\text { P-valor* }\end{array}$} \\
\hline & $\mathrm{N}$ & $\%$ & $\mathrm{~N}$ & $\%$ & & \\
\hline \multicolumn{7}{|l|}{ Condições Oferecidas } \\
\hline \multicolumn{7}{|l|}{ Escolaridade } \\
\hline Sem escolaridade e fundamental incompleto & 234 & 55,5 & 188 & 44,5 & 1,0 & \\
\hline Fundamental completo e médio incompleto & 166 & 60,1 & 110 & 39,9 & 1,21 & \\
\hline M édio completo & 136 & 62,1 & 83 & 37,9 & 1,32 & \\
\hline Superior incompleto e completo & 13 & 65,0 & 7 & 35,0 & 1,49 & \\
\hline Total & 549 & 58,6 & 388 & 41,4 & & $\begin{array}{l}\chi^{2}=3,3 \\
p=0,07\end{array}$ \\
\hline \multirow{2}{*}{\multicolumn{7}{|c|}{$\begin{array}{l}\text { Trabalho dos Profissionais } \\
\text { Escolaridade }\end{array}$}} \\
\hline & & & & & & \\
\hline Sem escolaridade e fundamental incompleto & 113 & 26,8 & 309 & 73,2 & 1,0 & \\
\hline Fundamental completo e médio incompleto & 64 & 23,2 & 212 & 76,8 & 0,83 & \\
\hline M édio completo & 46 & 21,0 & 173 & 79,0 & 0,73 & \\
\hline Superior incompleto e completo & 8 & 40,0 & 12 & 60,0 & 1,82 & \\
\hline Total & 231 & 24,7 & 706 & 75,3 & & $\begin{aligned} \chi^{2} & =0,91 \\
p & =0,34\end{aligned}$ \\
\hline
\end{tabular}

${ }^{*}$ P referente ao teste qui-quadrado de Tendência e $5 \%$ de significância

Tabela 5. Avaliação das questões de avaliação da qualidade do atendimento nas Unidades de Saúde da Família do Recife, 2009.

\begin{tabular}{|c|c|c|c|c|}
\hline \multirow{3}{*}{ Dimensões da qualidade } & \multicolumn{4}{|c|}{ Avaliação } \\
\hline & \multicolumn{2}{|c|}{ Insatisfeito } & \multicolumn{2}{|c|}{ Satisfeito } \\
\hline & $\mathrm{N}$ & $\%$ & $\mathrm{~N}$ & $\%$ \\
\hline Acessibilidade & 405 & 43,1 & 534 & 56,9 \\
\hline Dignidade e Cortesia & 329 & 35,0 & 610 & 65,0 \\
\hline Conforto & 424 & 45,2 & 515 & 54,8 \\
\hline Tempo de Espera & 630 & 67,1 & 309 & 32,9 \\
\hline Assistência Clínica ao U suário & 88 & 9,4 & 850 & 90,6 \\
\hline Confidencialidade das Informações & 79 & 8,4 & 859 & 91,6 \\
\hline Direito à Informação & 69 & 7,4 & 867 & 92,6 \\
\hline Atividades Educativas e Comunitárias & 549 & 58,5 & 390 & 41,5 \\
\hline
\end{tabular}

As questões que mais influenciaram na insatisfação com o conforto foram a quantidade e 0 conforto das cadeiras $(54,2 \%)$ e a temperatura da unidade $(46,1 \%)$. As questões com satisfação superior a $70 \%$ foram a limpeza da unidade e a conservação dos equipamentos.

Em relação aos tempos de espera para ser atendido e para receber o resultado dos exames, as unidades apresentaram uma alta insatisfação, $54,7 \%$ e $63,0 \%$, respectivamente.

As questões referentes à qualidade da assistência clínica receberam el evados percentuais de satisfação. D estacam-se valores superiores a 90\% as questões relacionadas à paciência do médico/ enfermeiro para escutar com atenção os problemas de saúde e o cuidado e detalhamento do médico/enfermeiro ao exame. A satisfação com a resolução de seu problema de saúde na consulta realizada foi de $78,6 \%$.

0 direito e a confidencialidade das informações, como visto anteriormente, tiveram grande satisfação dos usuários. As questões relacionavam-se às informações repassadas pelo médico/ enfermeiro sobre o problema de saúde e o tratamento $(92,6 \%)$ e a confiança no médico/enfermeiro para contar seus problemas de saúde $(91,6 \%)$.

Por fim, na dimensão atividades educativase comunitárias as questões que apresentaram maior insatisfação foram a possibilidade de participação de atividades educativas no momento de espera da consulta (73,9\%). Além disso, 51\% dos entrevistados estavam insatisfeitos com a visita domiciliar do médico em caso de necessidade. No entanto, 63,3\% dos entrevistados demonstraram-se satisfeitos com a orientação dos Agentes Comunitários de Saúde quanto ao uso adequado dos serviços de saúde durante as visitas domiciliares.

\section{Discussão}

Os usuários das USF têm como perfil predominante serem do sexo feminino, jovens e com baixa escolaridade. 0 alto número de mulheres de- 
monstra a importância delas como usuárias das USF e como cuidadoras da saúde de sua família. Resultados semelhantes foram encontrados em outros estudos ${ }^{16,17}$.

A menor participação dos homens deve ser decorrente por um lado, de um maior vínculo com o trabalho nesta faixa etária produtiva, buscando assim, outras alternativas para a assistência, ou por outro lado, pela não priorização a esses usuários

Em relação à vinculação dosusuários atendidos com a USF, demonstrada através da alta procura quando apresentam problemas de saúde, é um resultado muito bom eevidencia a Estratégia da Saúde da Família como porta de entrada do sistema de saúde. Esta vinculação da família com a USF é reforçada quando se observa que a grande mai oria dos residentes dos domicílios usam a unidade regularmente. Além disso, corrobora com o alto percentual de usuários que afirmaram terem seu problema de saúde resolvido na consulta realizada, dados também encontrados no estudo de Cotta et al. ${ }^{12}$.

Starfield ${ }^{18}$ afirma, que para funcionar como porta de entrada, a USF, além de ser acessível, deve constituir-seem serviço deprocura regular eservir de filtro e ponto de entrada do fluxo pela rede assistencial na busca coordenada pelo generalista.

Quanto à forma de agendamento da consulta, o percentual de quase $23 \%$ demarcação realizada pelos ACS pode evidenciar uma grande re solutividade da ação destes profissionais quando identificam problemas de saúde na comunidade, porém, podetambém demonstrar um desvirtuamento da prática realizada pelos ACS de promoção e prevenção em saúde.

A predominância dos atendimentos realizadosatravés de agendamento anterior, assim como a maioria dos atendimentos sendo realizados em até 7 dias e a quase totalidade em até 30 dias, demonstram a assistência a uma demanda programada aos grupos e programas específicos, assim como, o atendimento das intercorrências no mesmo dia. Estes resultados estão próximos dos parâmetros propostos por Starfield ${ }^{18}$, que estabelece que em $90 \%$ dos casos as urgências devem ser atendidas dentro de uma hora; os quadros agudos devem demorar um dia para conseguir consulta; e a rotina, os seguimentos, têm de ser agendados para uma semana.

Como os usuários foram entrevistados após 0 atendimento na USF, o alto percentual de níveis de saúde satisfatórios encontrados, sugere quea busca pelo atendimento não ocorreapenas a partir da doença instalada, mas a uma assis- tência à demanda programada na busca pela prevenção em saúde.

A alta satisfação com o trabalho dos profissionais é um importante resultado para a Estratégia da Saúde da Família que tem no trabalho dos profissionais e na sua relação com a população o elemento primordial de sua legitimação na organização do sistema de saúde, resultado semelhante aos encontrados por Cotta et al. ${ }^{12} \mathrm{e}$ Traverso-Yepez e M orais ${ }^{19}$, onde evidenciaram que o trabalho dos profissionais é o componente melhor avaliado pelos usuários.

A alta insatisfação com as condições oferecidas evidenciam condições das unidades de saúde insatisfatórias, condições que são fundamentais para implementação das ações e que permitem a consolidação da Estratégia da Saúde da Família. Para o M inistério da Saúde, apesar da Estratégia da Saúde da Família demonstrar melhora na eficiência e na qualidade dos serviços prestados na atenção básica, constata-se um número significativo de unidades com estrutura física inadequada, não raro, improvisada ${ }^{20}$.

Quanto maior a escolaridade, maior a insatisfação com as condições oferecidas nas unidades de saúde da família, o que demonstra uma maior exigência destes, por outro lado, a menor insatisfação dos com maior idade evidencia uma mai or complacência e uma maior exigência dos mais jovens.

Estes resultados se assemelham ao encontrado por Silva et al. ${ }^{16}$, que referem que os usuários com até 25 anos, têm probabilidade maior de estarem insatisfeitos com o PSF. Além disso, $M$ endes $^{21}$, em estudo nas emergências, também encontrou mais insatisfação nos usuários com maior escolaridade.

Dentre as dimensões da qualidade, foi observado que três delas apresentaram percentual de satisfação maior que $90 \%$, assistência clínica, confidencialidadee direito a informações, dimensões relacionadas à relação profissional-usuário, que tornam o atendimento solidário e humano, fortalecem a responsabilidade com as necessidades da comunidade e consolidam as premissas da Estratégia. Resultado semelhante foi encontrado no estudo realizado por Jorge et al. ${ }^{11}$, onde se chegou a conclusão de que os aspectos do processo, que envolvem o fator humano da relação médico-paciente, tais como o poder de esclarecimento do profissional, acolhimento, confiança e respeito são os determinantes da qualidade do PSF, e a eles são atribuídos maior ênfase.

A maior insatisfação com os tempos de espera, relativos ao agendamento de consulta especializa- 
da e realização de consulta na unidade, pode evidenciar dificuldades relacionadasà organização do serviço de saúde e à garantia de acesso à atenção especializada em tempo oportuno eresolutivo.

Estes dados, destacam semel hança aos resultados encontrados por Swarcwald et al. ${ }^{22}$, na Pesquisa Mundial de Saúde, no Brasil. Para estes autores, os aspectos relacionados à avaliação do atendimento ambulatorial, revelaram queo "tempo de espera até ser atendido" teve o menor grau de satisfação por parte dos usuários, dentre todos os aspectos analisados.

É importante destacar ainda, o elevado nível de insatisfação das dimensões acessibilidade e desenvolvimento de atividades comunitárias, intimamente relacionadas ao al cance dos objetivos da Estratégia de Saúde da Família.

$\mathrm{N}$ a dimensão da acessibilidade, embora os usuários tenham realizado uma boa avaliação quanto a distância da sua casa até a unidade de saúde, a avaliação da dimensão ficou prejudicada pela grandeinsatisfação com a referência para consulta especializada e a marcação de consulta na unidade. Percebe-se que a insatisfação refere se a um dos principais objetivos da unidade de saúde da família, porta de entrada para uma rede de serviços resolutivos de acesso universal.

Esta dificuldade de acesso aos serviços de referência da USF, équeixa frequente entres os usuários em todo o país também evidenciada nos estudos realizados por Teixeir ${ }^{23}$, Shimizu et al. ${ }^{24}$ e Souza et al. ${ }^{25}$.

A insatisfação com questões relativas ao de senvolvimento deatividades educativase comunitárias evidenciadas neste estudo demonstram dificuldades na implementação das estratégias básicas preconizadas para a ESF. A insatisfação com a visita dos médicos em caso de pessoas acamadas na residência éuma evidência das dificuldades, esta importância foi também constatada em estudo realizado por $M$ andú et al. ${ }^{26}$ que os usuários consideram a visita domiciliar do médico, como um meio de evitar que aquele indivíduo que tem uma dada necessidade especial precise se deslocar até a USF para ser atendido. Outra questão preocupante éa existência de um grau razoável de insatisfação com o trabalho dos ACS, resultado diferente de outras pesqui sas ${ }^{12,13}$ que apresentam boas avaliações pelos usuários.

A boa avaliação das dimensões dignidade $e$ cortesia no atendimento, confidencialidade edireito à informação, com exceção da possibilidade de fazer reclamações e ser ouvido que foi mal avaliado, além da satisfação com a assistência clínica ao usuário, como tratam da ação da equipe da USF, são coerentes com a boa avaliação do trabalho dos profissionais. A insatisfação com a possibilidade defazer reclamações sugere dificuldade em admitir o usuário como ator fundamental para o processo de avaliação dos serviços prestados pelas unidades.

Resultado semelhantefoi encontrado na avaliação da qualidade da assistência de urgência, onde foi evidenciado que os fatores que mais contribuem para uma maior satisfação dos usuários são referentes à ação dos profissionais de saúde ${ }^{17}$. Também na Pesquisa M undial de Saúde, no Brasi ${ }^{22}$, as questões que obtiveram melhor satisfação dos usuários referiam-se a aspectos que dizem respeito à intimidade, ao sigilo das informações pessoais e as habilidades dos profissionais de saúde.

A diversidade deresultadosapresentadosneste trabalho foi possível porque buscou avaliar as equipes de saúde da família, enfocando cada momento do atendimento, captando deste modo a percepção crítica dos usuários e evitando os vieses que levam à alta satisfação. Isto permite avançar na prática de avaliação no cotidiano da gestão em saúde, visando instituir mudanças na perspectiva dos princípios norteadores da atenção básica e no fortalecimento do Sistema Ú nico deSaúde, tomando o usuário como o objeto central do processo de trabalho. 


\section{Colaboradores}

RF Santiago trabal hou na concepção, coleta e sistematização de dados, delineamento e elaboração do texto. ACG M endes trabalhou na concepção, coleta e sistematização de dados, delineamento e elaboração do texto. GM D M iranda trabalhou na concepção, coleta e sistematização de dados, delineamento e elaboração do texto. BM ASM Furtado trabalhou na concepção, coleta e sistematização de dados. PO Duarte trabaIhou na concepção, coleta e sistematização de dados. WV Souza trabalhou na elaboração da metodologia.

\section{Referências}

1. César CLG, Tanaka OY. Inquérito domiciliar como instrumento de avaliação de serviços de saúde: um estudo de caso na região sudoeste da área metropolitana de São Paulo, 1989-1990. Cad Saude Publica 1996; 12(Supl. 2):59-70.

2. Minayo MCS. Conceito de avaliação por triangulação de métodos. In: M inayo M CS, Assis SG, Souza ER, organizadores. Avaliação por triangulação de métodos. Rio de Janeiro: Fiocruz; 2005.

3. Deslandes SF. Concepções em pesquisa social: articulações com o campo da avaliação em serviços de saúde. Cad Saude Publica 1997; 13(1):103-107.

4. Peixoto M PB. Perspectivas do Controle e Avaliação no Processo de Construção do SUS. In: Brasil. Secretaria de Políticas de Saúde. Gestão M unicipal de Saúde: textos básicos. Brasília: M S; 2001.

5. Donabedian A. The quality of care. How can it be assessed? JAM A 1988; 26(12):1743-1748.

6. Donabedian A. Explorations in Quality Assessment and M onitoring: The Definition of Quality and Approaches to its Assessment. Ann Harbor: Michigan H. A.; 1980.

7. M alik AM, Schiesari LM C. Q ualidade na Gestão Local de Serviços e Ações de Saúde. São Paulo: Faculdade de Saúde Publica, USP; 1998. (Série Saúde \& Cidadania, v. 3).

8. Acurcio FA, Cherchiglia ML, Santos M A. Avaliação de Qualidade de Serviços de Saúde. Saúde debate 1991; 33:50-53.

9. Fundação Oswaldo Cruz (Fiocruz). Projeto-Desenvolvimento de metodologia de avaliação do desempe nho do sistema de saúde brasileiro (PRO-ADESS): Relatório final. Rio de Janeiro: Fiocruz; 2003.

10. Felisberto E. Monitoramento e avaliação na atenção básica: novos horizontes. Rev. bras. saúde matern. infant. 2004; 4(3):317-321

11. Jorge MSB, Guimarães JMR, Vieira LB, Paiva FDS, Silva DR, Pinto AGA. Avaliação da qualidade do PSF no Ceará: a satisfação dos usuários. Rev. baiana saúde pública 2007; 31(2):256-206.

12. Cotta RM M, Marque ES, M aia TM, Azeredo CM, Schott M, Franceschini SCC, Priori SE. A satisfação dos usuários do Programa de Saúde da Família: avaliando o cuidado em saúde. Sci. med. 2005; 15(4):227-234.

13. Ronzani TM, Silva CM . O Programa de Saúde da Família segundo profissionais de saúde, gestores e usuários. Cien Saude Colet 2008; 13(1):23-34.

14. Gea MT, Hernán-García M, Jimenéz-M artín JM , Cabrera A. Opinión de los usuarios sobre la calidad del Servicio de Urgencias del Centro M édicoQuirúrgico Del Hospital Virgen de las Nieves. Rev. calid. Asist 2001; 16:37-44

15. Kirkwood BR. Essentials of medical statistics. Oxford: Blackwell; 2003.

16. Silva JA, Ribeiro LC, Silveira M. Avaliação do nível de satisfação dos usuários do Programa de Saúde da Família no Bairro Furtado M enezes - Juiz de Fora/M G. In: Encontro Nacional de Estudos Populacionais - ABEP, XIV, Caxambú; 2004. 
17. Mendes ACG. A delicadeza esquecida: Avaliação da qualidade das Emergências. Recife: Editora Universitária da UFPE; 2010. 296p.

18. Starfield B. Atenção Primária: equilíbrio entre necessidades de saúde, serviços e tecnologia. Brasília: Unesco, M S; 2002.

19. Traverso-Yepez M, Morais NA. Reivindicando a subjetividade dos usuários da Rede Básica de Saúde: para uma humanização do atendimento. Cad Saude Publica 2004; 20(1):80-88.

20. Brasil. M inistério da Saúde (M S). Secretaria de Atenção à Saúde. Departamento de Atenção Básica. $M$ anual de estrutura física das unidades básicas de saúde: saúde da família. 2ª Edição. Brasília: MS; 2009. (Série A. Normas e M anuais Técnicos)

21. Mendes ACG. Avaliação da Qualidade da Assistência de U rgência e Emergência: uma abordagem por triangulação de métodos [tese]. Recife (PE): Centro de Pesquisas Aggeu M agalhães, Fundação Oswaldo Cruz; 2009.

22. Szwarcwald CL, Leal MC, Gouveia GC, Souza WV. Desigualdades socioeconômicas em saúde no Brasil: resultados da Pesquisa Mundial de Saúde. Rev. bras. saúde matern. infant. 2005; 5(Supl. 1):1-22.

23. Teixeira SA. Avaliação dos usuários sobre o Programa de Saúde da Família em Vitória da Conquista - Bahia - Brasil. In: Projeto de desenvolvimento de sistemas e serviços de saúde. Braślia: OPAS; 2004.

24. Shimizu HE, Rosales $C$. A atenção à saúde da família sob a ótica do usuário. Rev. latinoam. enferm. [artigo original online]. 2008 set-out [acessado em 2012 fev 01]; 16(5): [cerca de 7 páginas]. Disponível em: http://www.scielo.br/pdf/rlae/v16n5/pt_14.pdf.

25. Souza ECF, Vilar RLA, Rocha NSPD, Uchoa AC Rocha PM. Acesso e acolhimento na Atenção Básica: uma análise da percepção do usuário e profissionais de saúde. Cad Saude Publica 2008; 24(Supl. 1):100-110.

26. Manduì ENT, Gaíva MAM, Silva MA, Silva AMN Visita domiciliária sob o olhar de usuários do Programa de Saúde da Família. Texto \& contexto enferm. 2008; 17(1):131-140.

Artigo apresentado em 08/11/2011

Aprovado em 29/03/2012

Versão final apresentada em 12/04/2012 